\title{
Accessory Nerve Disorder, CTCAE
}

National Cancer Institute

\section{Source}

National Cancer Institute. Accessory Nerve Disorder, CTCAE. NCI Thesaurus. Code C143258.

A disorder characterized by dysfunction of the accessory nerve (eleventh cranial nerve). 